\title{
Labyrinthe
}

40 | 2013

Comme les abeilles

\section{La proximité entre l'homme et l'abeille : un point de vue poétique (Maurice Maeterlinck)}

Laetitia Mouze

\section{(2) OpenEdition}

1 Journals

Édition électronique

URL : http://journals.openedition.org/labyrinthe/4301

DOI : $10.4000 /$ labyrinthe.4301

ISSN : 1950-6031

Éditeur

Hermann

Édition imprimée

Date de publication : 1 mars 2013

Pagination : 27-31

ISBN : 9782705688400

\section{Référence électronique}

Laetitia Mouze, « La proximité entre l'homme et l'abeille : un point de vue poétique (Maurice

Maeterlinck) », Labyrinthe [En ligne], 40 | 2013, mis en ligne le 01 mars 2015, consulté le 20 avril 2019.

URL : http://journals.openedition.org/labyrinthe/4301; DOI : 10.4000/labyrinthe.4301

Propriété intellectuelle 


\title{
La proximité entre l'homme et l'abeille : un point de vue poétique (Maurice Maeterlinck)
}

\author{
Laetitia Mouze
}

On peut tracer un parallèle entre Platon et Maeterlinck sur le point suivant: dans le Théétète, Platon fait de l'étonnement la mère de la philosophie. Le terme grec, thauma, signifie plus exactement « la merveille», « le prodige ». Cela peut être mis en rapport avec la récurrence, dans La vie des abeilles de Maeterlinck, du terme d' « admiration ». Dans l'ouvrage, sans cesse, Maeterlinck dit que les abeilles sont admirables, qu'elles suscitent de l'admiration: du point de vue de l'étymologie, le terme d' «admiration » est très proche du thauma de Platon. La Vie des abeilles est un ouvrage de sentiment: un témoignage d'admiration sur la vie des abeilles.

Parler de l' « anthropomorphisme » de Maeterlinck dans son approche des abeilles, c'est formuler un reproche : celui de parler en termes humains de quelque chose qui ne l'est pas, et dont on considère que d'en parler en termes humains revient à plaquer sur lui quelque chose qui ne lui convient pas, lui prêter des comportements, des intentions qui sont propres à l'homme. Dans La Vie des abeilles, c'est une pratique continuelle : l'apothéose est atteinte au moment où Maeterlinck évoque l'accouplement de la reine des abeilles, qui a lieu dans le ciel. Dans des pages d'un lyrisme échevelé, il parle de vol nuptial, de noces tragiques, d'étreinte, d'hymen. On comprend l'énervement du scientifique à la lecture de ces passages, qui ont pu discréditer aux yeux de gens sérieux - ou considérés comme tels - l'ouvrage de Maeterlinck.

Mais il faut aussi réfléchir à cet anthropomorphisme, à sa portée et à son sens: juste après la description de la noce de la reine, on trouve l'inverse, soit une description purement mécanique et matérielle de ce qui se passe dans le ciel lors de ces «noces tragiques ». Maeterlinck nous livre même les secrets de ces noces - pourquoi elles se passent dans le ciel, etc. Il enchaîne donc le morceau de bravoure lyrique avec 


\section{Labyrinthe, $n^{\circ} 40$}

la description matérialiste et mécanique, et il le fait, de surcroît, d'une manière qui, sans discréditer la description lyrique qu'il vient de faire, montre qu'il a bien conscience de l'excès de cette dernière, si éloignée de ce qui se passe réellement. Maeterlinck est parfaitement conscient du reproche d'anthropomorphisme qu'on peut lui faire, mais, à ce moment-là précisément, il le déjoue; comme s'il avait une légère ironie sur le texte très lyrique qu'il vient de donner. Il prend en tout cas une certaine distance avec cette description tragique. Ailleurs, il évoque l'anthropomorphisme comme une nécessité :

Puisqu'il nous est impossible de pénétrer la pensée des abeilles, il faut bien que nous interprétions humainement le spectacle auquel nous assistons.

On n'a pas le choix : et effectivement nous sommes des hommes, et il nous est difficile de parler des choses autrement qu'en hommes. Dans le livre II, qui porte sur l'essaim, au chapitre 21, Maeterlinck explique en substance que l'anthropomorphisme n'est qu'une manière de parler. Il ne dit pas les choses telles qu'elles sont, sachant qu'il n'y a pas d'autre manière de faire que d'avoir recours à cette manière de parler :

On se dira que ce sont là des conjectures bien hasardeuses et trop humaines, que les abeilles n'ont probablement aucune idée de ce genre, et que la notion de l'avenir, de l'amour de la race, et tant d'autres que nous leur attribuons, ne sont au fond que les formes que prennent pour elles la nécessité de vivre, la crainte de la souffrance et de la mort et l'attrait du plaisir. J'en conviens; tout cela, si l'on veut, n'est qu'une manière de parler, aussi n'y attaché-je pas grande importance. La seule chose certaine ici, comme elle est la seule chose certaine dans tout ce que nous savons, c'est que l'on constate que dans telle et telle circonstance, les abeilles se conduisent envers leur reine de telle ou telle façon. Le reste est un mystère autour duquel on ne peut faire que des conjectures plus ou moins agréables, plus ou moins ingénieuses.

Et Maeterlinck enchaîne sur quelque chose qui paraît capital, parce qu'il renverse l'accusation d'anthropomorphisme: 


\section{La proximité entre l'homme et l'abeille}

Mais si nous parlions des hommes, comme il serait peut-être sage de parler des abeilles, aurions-nous le droit d'en dire beaucoup davantage? Nous aussi nous n'obéissons qu'aux nécessités, à l'attrait du plaisir ou à l'horreur de la souffrance, et ce que nous appelons notre intelligence a la même origine et la même mission que ce que nous appelons instinct chez les animaux. Nous accomplissons certains actes, dont nous croyons connaître les effets, nous en subissons, dont nous nous flattons de pénétrer les causes mieux qu'ils ne font; mais outre que cette supposition ne repose sur rien d'inébranlable, ces actes sont minimes et rares, comparés à la foule énorme des autres, et tous, les mieux connus et les plus ignorés, les plus petits et les plus grandioses, les plus proches et les plus éloignés, s'accomplissent dans une nuit profonde où il est probable que nous sommes à peu près aussi aveugles que nous supposons que le sont les abeilles.

Il reprend un argument du même ordre à deux autres reprises, un peu en amont: il imagine un habitant de Mars ou de Vénus qui nous regarderait de loin, qui nous verrait nous agiter dans tous les sens, de façon pour lui absurde. Cet habitant d'ailleurs aurait bien du mal à nous prêter une intelligence, en nous voyant courir dans nos cités, un peu partout. Maeterlinck renverse de façon fine et intelligente l'accusation d'anthropomorphisme, en montrant qu'interpréter les actes des hommes en leur prêtant une intentionnalité et de l'intelligence, c'est peut-être trop leur prêter. Peut-être sommes-nous anthropomorphes non seulement quand il s'agit d'abeilles ou d'autres animaux, mais aussi, et surtout, quand nous parlons des hommes. 
Remontons aux noces tragiques de la reine. Dans l'exemple qui nous occupe, la nature veut donc, en vue de la fécondation croisée, que l'accouplement du faux-bourdon et de la reine abeille ne soit possible qu'en plein ciel. Mais ses désirs se mêlent comme un réseau et ses lois les plus chères ont à passer sans cesse à travers les mailles d'autres lois, qui l'instant d'après passeront à leur tour à travers celles des premières.

Ayant peuplé ce même ciel de dangers innombrables, de vents froids, de courants, d'orages, de vertiges, d'oiseaux, d'insectes, de gouttes d'eau qui obéissent aussi à des lois invincibles, il faut qu'elle prenne des mesures pour que cet accouplement soit aussi bref que possible. II l'est, grâce à la mort foudroyante du mâle. Une étreinte y suffit, et la suite de l'hymen s'accomplit aux flancs mêmes de l'épouse.

Celle-ci, des hauteurs bleuissantes, redescend à la ruche tandis que frémissent derrière elle, comme des oriflammes, les entrailles déroulées de l'amant. Quelques apidologues prétendent qu'à ce retour gros de promesses, les ouvrières manifestent une grande joie. Büchner, entre autres, en trace un tableau détaillé. J'ai guetté bien des fois ces rentrées nuptiales et j'avoue n'avoir guère constaté d'agitation insolite, hors les cas où il s'agissait d'une jeune reine sortie à la tête d'un essaim et qui représentait l'unique espoir d'une cité récemment fondée et encore déserte. Alors toutes les travailleuses sont affolées et se précipitent à sa rencontre. Mais pour l'ordinaire, et bien que le danger que court l'avenir de la cité soit souvent aussi grand, il semble qu'elles l'oublient. Elles ont tout prévu jusqu'au moment où elles permirent le massacre des reines rivales. Mais arrivé là, leur instinct s'arrête ; il y a comme un trou dans leur prudence. Elles paraissent donc assez indifférentes. Elles lèvent la tête, reconnaissent peut-être le témoignage meurtrier de la fécondation, mais encore méfiantes, ne manifestent pas l'allégresse que notre imagination attendait. Positives et lentes à l'illusion, avant de se réjouir, elles attendent probablement d'autres preuves. On a tort de vouloir rendre logiques et humaniser à l'extrême tous les sentiments de petits êtres si différents de nous. Avec les abeilles, comme avec tous les animaux qui portent en eux un reflet de notre intelligence, on arrive rarement à des résultats aussi précis que ceux qu'on décrit dans les livres. Trop de circonstances nous demeurent inconnues. Pourquoi les montrer plus parfaites qu'elles ne sont, en disant ce qui n'est pas ? Si quelques-uns jugent qu'elles seraient plus intéressantes si elles étaient pareilles à nous-mêmes, c'est qu'ils n'ont pas encore une idée juste de ce 
qui doit éveiller l'intérêt d'un esprit sincère. Le but de l'observateur n'est pas d'étonner, mais de comprendre, et il est aussi curieux de marquer simplement les lacunes d'une intelligence et tous les indices d'un régime cérébral qui diffère du nôtre, que d'en rapporter des merveilles.

Pourtant, l'indifférence n'est pas unanime, et lorsque la reine haletante arrive sur la planchette d'abordage, quelques groupes se forment et l'accompagnent sous les voûtes, où le soleil, héros de toutes les fêtes de la ruche, pénètre à petits pas craintifs et trempe d'ombre et d'azur les murailles de cire et les rideaux de miel. Du reste, la nouvelle épousée ne se trouble pas plus que son peuple, et il n'y a point place pour de nombreuses émotions dans son étroit cerveau de reine pratique et barbare. Elle n'a qu'une préoccupation, c'est de se débarrasser au plus vite des souvenirs importuns de l'époux qui entravent sa démarche. Elle s'assied sur le seuil, et arrache avec soin les organes inutiles, que des ouvrières emportent à mesure et vont jeter au loin ; car le mâle lui a donné tout ce qu'il possédait et beaucoup plus qu'il n'était nécessaire. Elle ne garde, dans sa spermathèque, que le liquide séminal où nagent les millions de germes qui, jusqu'à son dernier jour, viendront un à un, au passage des œufs, accomplir dans l'ombre de son corps l'union mystérieuse de l'élément mâle et femelle dont naîtront les ouvrières. Par un échange curieux, c'est elle qui fournit le principe mâle, et le mâle le principe femelle. Deux jours après l'accouplement, elle dépose ses premiers œufs, et aussitôt le peuple l'entoure de soins minutieux. Dès lors, douée d'un double sexe, renfermant en elle un mâle inépuisable, elle commence sa véritable vie, elle ne quitte plus la ruche, ne revoit plus la lumière, si ce n'est pour accompagner un essaim; et sa fécondité ne s'arrête qu'aux approches de la mort.

Maurice Maeterlinck, La Vie des abeilles, livre V, « Le vol nuptial », chapitre IX. 